\title{
Manejo Quirúrgico de las Alteraciones Mamarias
}

\author{
(Displasia, Tumores, Hiper e Hipo Mastias, Ptosis y Asimetr las)
}

Dres.: Fernando del Corral*, Augusto Botero T.**

En el examen integral de la mujer, es el ginecológo quien tiene la mejor oportunidad de practicar una revisión cuidadosa de los senos. Por ello es a él a quien corresponde adquirir una completa formación para el manejo apropiado de las alteraciones de la glándula mamaria. Queremos presentar nuestra experiencia con el manejo de las alteraciones patológicas del seno, en un grupo de pacientes de nuestra clientela privada. Mostramos la frecuencia y la asociación entre sí de las diferentes entidades patológicas. Pretendemos hacer un llamado importante a la "Displasia Mamaria" por su estrecha relación con el carcinoma mamario. Hemos agrupado dentro de las "Displasias" a la adenosis, con la enfermedad fibrosa y quística de la glándula, por ceñirse extrictamente a un mismo proceso etiológico, evolutivo y de manejo.

\footnotetext{
* Presidente Honorario, Sociedad Colombiana de Médicos Endoscopistas.

** Miembro Fundador Centro Integral de Fertilidad y Esterilidad.
}

Hemos sido consultados con mucha frecuencia fuera del aspecto propiamente médico, sobre aspectos estétitos y cosméticos. Por ello creemos que este factor altamente deseado por la mujer, debe ser tenido en cuenta y creemos que es obligación del especialista preservar los derechos de su paciente. Presentamos las correcciones cosméticas prácticadas, tales como: Manejo de la aplasia, hiper e hipomastias, asimetría, ptosis y deformidades quirúrgicas post-mastectomía.

Creemos necesario hacer una descripción de estos procedimientos y los resultados por nosotros obtenidos a corto y largo plazo.

\section{MATERIAL Y METODOS}

Se estudiaron 126 pacientes de consulta privada, sometidas a diferentes procedimientos quirúrgicos sobre el seno. En estas pacientes se encontraron seis entidades diferentes, habiendo pacientes con más de una alteración uni o bilateral, lo que nos da un total de 141 casos que se dividieron en dos grupos a saber: 
a) Alteraciones Clínicas

b) Alteraciones Estéticas

El grupo de las alteraciones clínicas se clasificó, de acuerdo con el resultado histo-patológico de la pieza obtenida en 106 casos, con los siguientes resultados:

Tabla No. 1

(ALTERACIONES CLINICAS)

\begin{tabular}{|c|c|c|}
\hline A. & TOTAL & $\%$ \\
\hline $\begin{array}{l}\text { DISPLASIA MAMARIA } \\
\text { (Enfermedad Fibroquistica) }\end{array}$ & 68 & 64.0 \\
\hline FIBROADENOMA & 27 & 25.5 \\
\hline PAPILOMA INTRADUCTAL & 4 & 3.8 \\
\hline CARCINOMA DUCTAL & 3 & 2.9 \\
\hline LIPOMA & 2 & 1.9 \\
\hline \multirow[t]{2}{*}{ CISTOSARCOMA PHILLODES } & 2 & 1.9 \\
\hline & 106 & 100.0 \\
\hline
\end{tabular}

b) Los 35 casos restantes, fueron considerados como alteraciones estéticas y se clasificaron como sigue:

Tabla No. 2

(ALTERACIONES ESTETICAS)

\begin{tabular}{|l|r|r|}
\cline { 2 - 3 } \multicolumn{1}{c|}{} & CASOS & $\%$ \\
\hline HIPOMASTIA & 16 & 45.7 \\
\hline PTOSIS & 11 & 31.4 \\
\hline HIPERPLASIA MAMARIA & 4 & 11.4 \\
\hline ASIMETRIA & 3 & 8.6 \\
\hline GLANDULAS SUPERNUMERARIAS & 1 & 2.9 \\
\hline TOTAL & 35 & 100.0 \\
\hline
\end{tabular}

Las pacientes del primer grupo se manejaron de acuerdo con el criterio clínico o radiológico practicando biopsia por congelación en los casos sospechosos de malignidad o en diferido de acuerdo con los hallazgos operatorios. Las pacientes fueron sometidas a diferentes procedimientos uni o bilateralmente, mediante resección tumoral, resección de cuadrante (Cuadrantopsia), mastectom ía simple subdérmica, simple total y mastectomía radical.

\section{Displasia Mamaria:}

Como en otras publicaciones, encontramos también que es la afección más frecuente de la patología del seno (64\%). Su mayor incidencia ocurrió en pacientes de la cuarta década $(38.2 \%)$, seguido en frecuencia en pacientes menores de 30 años y en mayores de 40 (30.9\%).

El diagnóstico se hizo por sospecha clínica en todos los casos los que fueron comprobados por biopsia por congelación o ecisión, verificando el diagnostico.

Se sometieron a los tratamientos convencionales que consistieron en:

Complejo B, Antiinflamatorios, Progestágenos $y$ nor esteroides orales en segunda mitad del ciclo y progestágenos de depósito (Depo-provera) cada 70 a 90 días. En pacientes mayores de 35 años se utilizaron además antigonadotróficos (Danazol) en 6 pacientes. Los resultados fueron muy pobres con el tratamiento médico y sólo se observó ligera mejoría, cuando además, del tratamiento médico la resección fue amplia (Cuadrantopsia). Las recurrencias fueron altas, teniendo que reintervenir 17 de 68 pacientes (25\%) en pacientes con tratamientos de 1 a 7 años. La incidencia de carcinoma en este grupo de pacientes con enfermedad fibroquística fue muy alta, del órden de 3.8 \%, lo que está de acuerdo con las cifras dadas por otros autores (1.2). Con frecuencia encontramos una estrecha relación entre la enfermedad fibroquística y los cuadros de hiper-estrogenismo, asociación con fibromatosis uterina (17.6\%) 
y alteraciones del ciclo con hipermenorrea. La vimos también asociada con adenomiosis y endometriosis, en 11.7\%, sin poder explicar dicha relación.

No encontramos asociación con multiparidad, lo que si vimos en relación con el fibroadenoma, más frecuente como es de esperar, en pacientes nulíparas.

\section{Adeno Fibroma:}

La encontramos como la segunda patología en frecuencia, 27 casos (25.5\%) y preponderamente en mujeres por debajo de 30 años (43.9\%). Los hallazgos fueron siempre clínicos con tumores con un promedio de $3 \mathrm{cms}$. de diámetro y generalmente unilaterales. En esta entidad a diferencia de la enfermedad fibroquística, el tratamiento fue únicamente quirúrgico, encontrando solamente un caso de recurrencia en una paciente que desarrolló un Cistosarcoma Phillodes.

En todos los casos se practicó una resección, un poco más liberal de la aconsejada, por la estrecha relación de esta entidad con el carcinoma, ya que en el $15 \%$ de los mismos provienen de adeno fibromas (2) y con el Cistosarcoma Phillodes que constituyen el 2\% de los adenomas.

Las correcciones se hicieron a toda costa, preservando la estética del seno con pequeñas incisiones, desplazadas a sitios de mayor conveniencia estética y siguiendo las lineas de Langer en la piel.

\section{Papiloma Intraductal:}

Observamos dentro de este grupo 4 casos. A diferencia del fibroadenoma, lo encontramos en 75\% por encima de los 40 años. La mayor parte presentaron secreción serosa o (sanguinolenta) por el pezón, lo que está de acuerdo con otras publicaciones (2). Es importante tomar citología de la secreción, por la técnica de Papanicolaou, sistemáticamente. En la clínica Mayo encontraron carcinoma en 57\% casos con secreción sero hemática.

En presencia o ausencia de tumor, estas pacientes fueron manejadas con incisión periareolar y resección canalicular amplia; solamente esta resección permite diferenciar la forma maligna de un papiloma intraductal (carcinoma papilar).

\section{COMENTARIOS}

Hemos anotado que la adenosis y la enfermedad fibrosa y quística son procesos evolutivos de una misma alteración endocrina de etiología no resuelta.

Sólo sabemos que en estas pacientes prevalece un hiper-estrogenismo absoluto o relativo, en el cual juega un papel preponderante el metabolismo estrogénico en el órgano afector.

La adenosis por sus características clínicas (tumor) se confunde con el carcinoma y solamente una biopsia puede excluir su malignidad. La enfermedad fibroquística es la más frecuente enfermedad del seno y la que mayor relación tiene con el carcinoma.

En las series del estado de Nueva York descritas por Haagensen (1) determinan que pacientes con enfermedad fibroquística son 3 a 4 veces más propensas a desarrollar carcinoma del seno.

En los casos del presente estudio, encontramos 3 pacientes con carcinoma (3.8\%) cuyo diagnóstico inicial fue de enfermedad fibro quística (uno de los casos bilateral).

Los procedimientos clínicos son insuficientes para el diagnóstico y aún la mamografía con sus diferentes técnicas no permite descartar una lesión maligna. 
Por lo tanto, sólo la biopsia dirigida o ampliada (cuadrantópsia) permite un adecuado diagnóstico. Una vez verificado el mismo y sólo en casos de recurrencia, la punción exploradora hecha sobre un nódulo de crecimiento $y$ supuestamente quístico, puede estar justificada.

Hemos visto reiteradamente los fracasos que se obtienen con los tratamientos médicos convencionales ya enumerados y solamente hemos obtenido alguna mejoría con cirugías ampliadas. Solamente 5 pacientes manejadas con progestágenos de depósito estuvieron asintomáticas, después de 3 años de tratamiento (7.5\%).

En los casos severos que con frecuencia llegan a la cancerofobia, estas pacientes son tributarias de la mastectomía subcutánea y aplicación de prótesis; por ello Ilegamos a la resección glandular completa en 4 pacientes (5.9\%).

Queremos señalar que el tratamiento de la enfermedad fibroquística del seno es actualmente junto con el de la endometriosis uno de los grandes retos de la ginecología moderna.

El tratamiento del fibroadenoma fue esencialmente quirúrgico y los resultados fueron satisfactorios. Solamente observamos 3 casos de Cistosarcoma Phillodes que consultaron primariamente por tumores deformantes y solamente uno con antecedentes de haber sido intervenida previamente por fibroadenoma. Estos son de altá tendencia a la malignidad.

En su componente estromal se encuentran elementos como tejido osteoide, cartílago o tejido lipoblástico y son generalmente de crecimiento rápido y características histólogicas bizarras, lo que lleva a ser tratado con amplia resección de tejido sano a su alrédedor.

\section{ALTERACIONES ESTETICAS}

Como mencionamos inicialmente, tenemos especial precaución en todos los casos en que se hizo necesaria una resección quirúrgica, en preservar al máximo posible la aparición estética de la glándula; para ello se siguieron las normas de cirugía estética y las incisiones se hicieron preferentemente sobre las líneas de Langer.

Al practicar la cuadrantopsia fue evidente el hecho de que al cerrar la brecha quirúrgica mejora considerablemente el aspecto cuneiforme del seno. De las alteraciones estéticas propiamente dichas, se manejaron un total de 35 pacientes que fueron clasificadas en: 16 casos de Hipomastia (45.7\%), 11 casos de Ptosis (31.4\%), 4 de Hiperplasia $(11.4 \%), 3$ de Asimetría (8.6\%) y un caso de mamas supernumerarias (2.9\%).

Los casos de Asimetría se manejaron con reducción o aumento contralateral con prótesis, de acuerdo con cada caso.

En los casos de Hipertrofia o Hipermastia se manejaron por reducción con corrección simultánea de la Ptosis, empleando la transposición de la areola según la técnica de Lexer (7) y modificaciones de Goldenberg (8).

Las Ptosis con hipomastia (11 casos) y la hipomastia per se (16 casos) fueron manejadas por un mismo procedimiento con utilización de prótesis mamaria. Por ello creemos necesario hacer una descrip. ción de los mismos y mostrar nuestros resultados. El material utilizado en sus diferentes modelos, corresponden en general a un mismo material mediante el uso de elastómeros de las siliconas.

Se aplicaron 27 prótesis, de las cuales 9 fueron semi-sólidas (Dow Corning) 
y las restantes, 18, fueron del tipo expandible mediante solución salina producidas por Heyer Shulte. No usamos prótesis mixtas tipo Hartley. El volúmen entre unas y otras osciló entre 100 a 350 cc de capacidad; en las prótesis usadas no se usaron aquellas con parches de fijación o plaquetas de celulosa.

Se llenaron los requisitos de esterilización y aplicación siendo múltiples sus ventajas.

Se tomaron en cuenta las contraindicaciones para su aplicación las cuales pueden ser resumidas en los siguientes puntos:

Figura No. 1

MASTECTOMIA SUB CUTANEA

Ent. Fibroquistica Recurrente Surco Sub-momorio

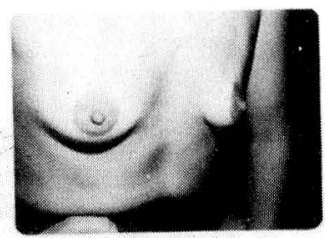

Antes

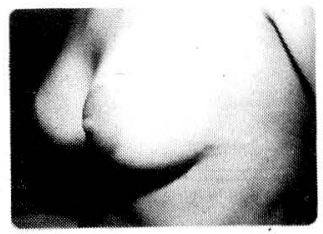

Después

Figura No. 2

PTOSIS MIPOMASTIA
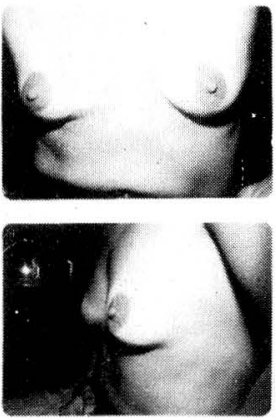

Antes
Surco Sub-mamatio
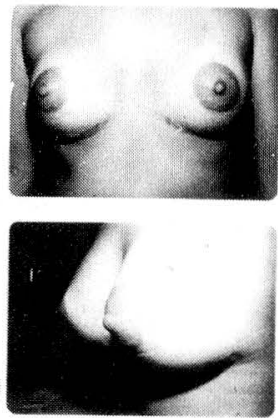

Después
Figura No. 3
HIPOMASTIA
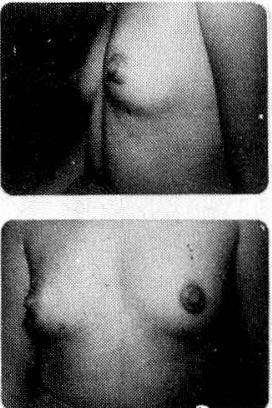

Antes
Insición Pora-areolar
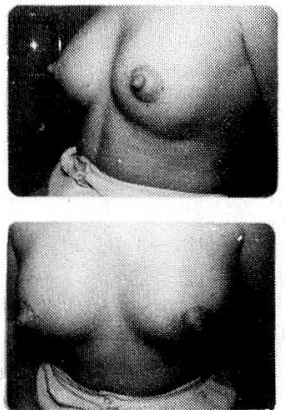

Despues
Historia reciente de infección, mastitis crónica dolorosa y difusa, presencia de tumor, Ptosis completa e historia familiar de cáncer del seno.

Sus complicaciones descritas por otros autores se pueden sumarizar en:

Firmeza exagerada por encasuplación (contractura fibrosa); formación de líquido excesivo (seroma o hematoma); necrosis del colgajo areola o mamelón; infección; complicaciones neurales tales como hiperestesia temporal o definitiva y finalmente, desplazamiento de la prótesis.

En nuestros casos, tuvimos fijeza e induración por fibrosis tardía en dos pacientes (7\%), ambas con prótesis semisólidas tipo (Dow - corning), de las cuales una fue manejada por capsulotomía; un caso de infección de la herida sin consecuencias ni expulsión de las prótesis y 2 casos de ruptura intraoperatoria de la prótesis, con remplazo inmediato.

Tuvimos 6 casos (22\%) de galacto rea copiosa en el post-operatorio no descritos por otros autores. Se manejaron con buen resultado con la administración de 5 mgrs. diarios alfa-bromo-ergocriptina 
durante un período de una a dos semanas.

Es importante anotar que guardados los requisitos de esterilización de las prótesis; preservando su técnica de aplicación y no contaminación (jabones, talco, etc.) los resultados son satisfactorios $\mathrm{y}$ corrigen estética y psicológicamente los problemas por los cuales fuimos consultados.

En los casos de contractura capsular su mejor tratamiento es prevenirla, mediante la aplicación sub-muscular de las prótesis (4) (5). Parece también eviden-

\section{SUMARIO}

Se estudiaron 126 pacientes sometidas a cirugía del seno. En ellas se encontraron seis entidades diferentes teniendo algunas pacientes más de una alteración uni o bilateral; para un total de 141 casos estudiados. Las alteraciones clínicas más frecuentes encontradas sobre 106 casos fueron, la Displasia mamaria 68 casos, $64 \%$ y el fibroadenoma con 27 casos, 25.5\%. Se hizo especial énfasis en el fracaso con el tratamiento médico de la displasia y en su gran tendencia a la recurrencia o perseverancia siendo necesario reintervenir quirúrgicamente 17 de 68 pacientes (25\%). Se encontró Carcino-

\section{ABSTRACT}

126 private patients who had breast surgery were studied. Six different entities were found and more than one patient had alterations in one or both breasts for 141 cases studied. The most frequent clinical alterations found in 106 cases were: Mammary displasia in 68 cases, $64 \%$ and fibroadenoma in 27 cases, $25.5 \%$. Special emphasis was done te que en las prótesis distendibles con solución salina, la contractura puede ser menor que con el uso de las sólidas (6); además, estas contracturas pueden ser previsibles evitando causas etiológicas tales como hematomas, seromas, infección y cuerpos extraños peri-capsulares (jabones, etc.). No obstante, existen comportamientos y reacciones fibrosas no previsibles propias de cada paciente.

Podemos decir que en general, quedamos satisfechos de nuestros resultados y por ello seguiremos insistiendo en este tipo de tratamientos.

ma en 3 casos (3.8\%), que con diagnóstico de Displasia, fueron sometidos a biopsia. Se sometieron a procedimientos quirúrgicos de alteraciones estéticas 35 pacientes. De ellas 27 se manejaron en Prótesis, 11 casos para ptosis e hipomastia y 16 casos con hipomastia sola. Las complicaciones fueron mínimas si se tienen en cuenta las indicaciones y las contraindicaciones. Las más sobresalientes fueron la galactorrea post-operatoria en 6 pacientes y la fijeza o fibrosis en dos casos. Se presentaron 2 rupturas intraoperatorias de la prótesis; una por probable defecto de fabricación y otra por accidente.

in the medical treatment failure and the great tendency to recurrence or perseverance, being necessary to operate again 17 patients from $68(25 \%)$. It was foundcarcinoma in three cases $3.8 \%$ with diagnosis of displasia previous biopsy. 35 patients had corrective surgery for esthetic alterations, 27 of them were given prosthesis. 11 cases were treated for ptosis and hipomastia and 16 cases just hipomastia. There was a minimum of 
complications if we think about the indications and counterindications. The most important were post-operative galactorrea in six patients and fibrosis in two cases. There were 2 intraoperatory ruptures of the prothesis; one of

\section{BIBLIOGRAFIA}

1. SQUARTINI, F., LOTTI, G., and BIANCIFIORI, C.: Fibroadenoma e cancro della mamella, Lav. d. Ist. ant e istol., Pat, Perugia, 13: 201, 1953.

2. HAAGENSEN C.D. Diseases of de Breast W.B. Saunders co. Phila. 167, 1956.

3. HOFFMAN S., the management of severe capsular contractures following breast augmentation Aesth, Plast. Surg Vol. 7 No. 2 109-112, 1983.

4. GRIFFITHS; Co, the sub muscular implant in augmentation mammaplasty. In trans Fourth Internat Cong Plast Surg, Amsterdarn: Excepta Medica Fourdation 1967. p - 1009.

5. PAPILLON J: Pros and cons of sub pectoral Implantation Clin Plast Surg 3: 320, 1976. them probably because of a faulty device and the uther one because of an accident.

Generally speaking we can say we are satisfied with our results so we will insist in this type of handling.

6. REIFFEL R.S., REES T.D. GUY C.L., ASTON CH. a comparison of Capsule Formation Following Breast Augmentation by Saline Filled or Gell-Filled Implants. Aesth. Plast. Surg 7: 113-116, 1983.

7. MALINIAC J.W., HARO GARCIA F. Deformidades mamarias y su tratamiento, 109, Editorial Labor S.A. 1952.

8. GOLDENBERG B. Comunicación personal Clínica Remedios, 1985. Cath, Co lombia.

\section{PARA SEPARATAS DIRIGIRSE A:}

Dr. Fernando del Corral. Clínica de los Re. medios. Oficina No. 325 Cali, Colombia. 